\title{
Estudo das queixas sobre saúde bucal em uma população de idosos na cidade de Ribeirão Preto-SP
}

Study on oral health complaints in an elderly population in Ribeirão Preto city, state of São Paulo, Brazil

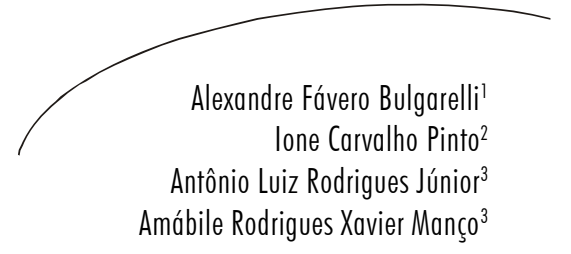

\section{Resumo}

O levantamento de queixas em saúde pode servir como indicador para se estudar necessidades de saúde de um determinado grupo de idosos. Neste contexto, o presente estudo descritivo transversal objetivou analisar as queixas de idosos relacionadas à saúde bucal mais prevalentes, associadas a idade, sexo e escolaridade. Utilizou-se uma amostra não-probabilística intencional de idosos adscritos na área de abrangência de um Núcleo de Saúde da Família na cidade de Ribeirão Preto/SP. Aplicou-se um questionário estruturado, através de entrevistas domiciliares, para o levantamento dos dados, os quais passaram por análise estatística bivariada. Os resultados mostraram maior representatividade do sexo feminino $(65,5 \%)$, com idade variando entre 60 e 69 anos (46,7\%), e baixa escolaridade. As frequências das queixas foram semelhantes para ambos os sexos. As queixas mais prevalentes foram mau hálito, boca seca e problemas referentes à articulação temporomandibular. Não foram encontradas associações significativas entre queixas e idade, porém houve associação significativa entre mau hálito e escolaridade $(\mathrm{p}=0,011)$. Os achados sugerem que a autopercepção frente às

\author{
Palavras-chave: \\ Necessidades e \\ Demandas de \\ Serviços de Saúde. \\ Idoso. Saúde Bucal. \\ Epidemiologia \\ Descritiva. Estudos \\ Transversais. \\ Análise Estatística. \\ Saúde do Idoso. \\ Queixas bucais. \\ Ribeirão Preto-SP
}

Universidade de São Paulo, Programa de Pós-Gradução Enfermagem em Saúde Pública, Escola de Enfermagem de Ribeirão Preto, Ribeirão Preto, SP, Brasil

2 Universidade de São Paulo, Escola de Enfermagem, Departamento de Saúde Materno Infantil e Saúde Pública. Ribeirão Preto, SP, Brasil

3 Universidade de São Paulo, Departamento de Medicina Social, Faculdade de Medicina de Ribeirão Preto. Ribeirão Preto, SP, Brasil

Correspondência / Correspondence

Alexandre Fávero Bulgarelli

Rua Ângelo Beloni, 253

14090-400 - Ribeirão Preto, SP, Brasil

E-mail: alefavbulg@usp.br 
condições de saúde bucal foram essenciais para o relato de algumas queixas, e que muito se tem a estudar frente às queixas de idosos, visto que muitas envolveram outros órgãos da cavidade bucal como articulações e glândulas.

\section{Abstract}

A survey about oral health complaints can be used as an indicator to study the health needs in a specific elderly group. In this context, the present crosssectional study aimed to evaluate the most frequent complaints of elderly concerning oral cavity conditions, and associated with age, gender and schooling. In an elderly population enrolled at a Family Health Nucleus, interviews were carried out with a structured household survey for data collection. A bivariate statistical analysis was conducted. The interviewed population was mostly represented by $65.5 \%$ of female elderly. Most of the population was 60 to 69 years old $(46.7 \%)$, with low schooling. There were almost the same frequencies of complaints in both sexes. The most frequent complaints were halitosis, dry mouth and problems related to temporomandibular joint. There was no statistical significance between complaints and age, but there was statistical significance association between halitosis and schooling $(p=0.011)$. The results suggested that self-perception of oral health conditions were essential to some oral health complaints, and there is a lot to study about this issue. This study showed that there was a large number of complaints of elders involving different oral organs as joints and glands.
Key words: Health Services Needs and Demand. Aged. Oral Health. Epidemiology, Descriptive. CrossSectional Studies. Statistical Analysis. Elderly's health. Oral complaints. Ribeirão Preto city-SP
INTRODUÇÃO

O envelhecimento da população brasileira é caracterizado pelo aumento do número de idosos na população, bem como o aumento da expectativa de vida dos mesmos. No Brasil, o avanço da medicina, a valorização da Atenção Primária à Saúde, o desenvolvimento de políticas públicas saudáveis voltadas aos idosos, e o novo paradigma da promoção da saúde, dentre outros inúmeros fatores, contribuem para que os indivíduos envelheçam saudáveis, atingindo idades cada vez mais avançadas. Já no início da década de 90 , projeções feitas pelo
IBGE mostraram que no Brasil, entre 2010 e 2020, a população de 65 anos alcançará uma taxa de crescimento de $3,8 \%$ ao ano, o que irá superar o próprio crescimento populacional. ${ }^{1}$ Assim, os desafios que surgem com o envelhecimento da população brasileira suscitam estudos com diferentes variáveis para o levantamento de novas hipóteses em pesquisas cientificas. ${ }^{2}$ Estes desafios são referentes aos avanços na ciência e tecnologia que permitirão ao idoso superar limites de forma independente e livre de doenças. ${ }^{3}$

A Política Nacional de Saúde Bucal estabelece, em seus princípios norteadores de 
ações, que a responsabilidade dos cirurgiões dentistas deve estar voltada também à disponibilidade de tempo para escuta das queixas em saúde bucal. ${ }^{4}$ Neste contexto, o cuidar em saúde, como relatado por alguns autores, reflete o desenvolvimento da capacidade de escuta em coletividade. Ao queixar-se, o indivíduo verbaliza um desconforto, um incômodo ou mesmo uma sintomatologia que, juntamente com os sinais de determinada patologia, acabam por auxiliar no diagnóstico de determinadas enfermidades. Esta verbalização gera, na maioria das vezes, a busca por conhecimento e informação dos cuidados e tratamentos com a saúde bucal. Estes aspectos evidenciam a importância de se produzir um vínculo que norteia as necessidades de saúde do indivíduo. ${ }^{5,6}$ Muito se tem a observar quando assuntos como queixas em saúde são estudados, visto que certos estudos que avaliam condições de saúde das pessoas não levam em consideração aspectos sociais e culturais que influenciam as queixas dos indivíduos ${ }^{\top}$ e, assim, é necessário conhecer a percepção dos indivíduos quanto à própria saúde bucal. É com esta ideia que se acredita na importância em ouvir os idosos a respeito deste aspecto, visto que o Brasil vive na atualidade um processo de reorganização, adequação e humanização dos serviços públicos de saúde embasados na Atenção Primária à Saúde.

Com o envelhecimento, os dentes e a gengiva passam por mudanças fisiológicas. Os dentes sofrem deslocamentos para compensar desgastes nas faces de contato de um com o outro. $O$ periodonto, tecido gengi- val e ósseo de sustentação dos dentes, talvez seja a estrutura mais afetada com a senescência, sendo que pelo próprio envelhecimento a gengiva sofre uma migração apical, que por sua vez pode levar à exposição da raiz do dente. Outras alterações, além das dentárias e gengivais, presentes na cavidade bucal, são referidas como queixas por pessoas idosas, e as mais frequentes envolvem outros órgãos como língua e glândulas salivares. A deficiência na secreção salivar, conhecida como xerostomia, é uma queixa muito comum por estar associada às doenças autoimunes, radioterapia e utilização de certos medicamentos. ${ }^{8,9}$ Muitas queixas estão associadas a estas alterações fisiológicas que ocorrem no organismo com o passar dos anos, e tais alterações na cavidade bucal, no entanto, podem ser controladas e prevenidas, o que deixa de lado a ideia de que envelhecimento significa contrair doenças bucais. A necessidade de mudança desta imagem negativa foi ressaltada na literatura, e profissionais da área da saúde devem ter cuidados especiais e diferenciados com o paciente idoso. ${ }^{10}$ Todavia, a odontologia deve fazer parte de um grupo interdisciplinar em saúde para garantir que as próximas gerações de idosos desfrutem de uma boa saúde bucal. ${ }^{11}$

Queixas relacionadas a problemas bucais relatadas por pacientes idosos não são objetos de estudo na atualidade. Assim, o presente estudo foi conduzido com o objetivo de levantar as principais queixas relatadas por idosos referentes às condições bucais, observando possíveis associações com algu- 
mas variáveis sociodemográficas, bem como contextualizar estes idosos frente à participação na pesquisa.

\section{MATERIAL E MÉTODO}

O presente estudo, quantitativo descritivo transversal, teve como população de referência (universo populacional) indivíduos com 60 anos ou mais de idade, de ambos os sexos, adscritos na área de abrangência do Núcleo de Saúde da Família III (NSF III), do Centro de Saúde Escola da Faculdade de Medicina de Ribeirão Preto da Universidade de São Paulo (CSE-FMRP-USP), totalizando 503 indivíduos, no início de 2006. Por se tratar de um estudo onde seria necessário haver certa interatividade entre entrevistado e pesquisador, confiabilidade dos dados e levantamento de queixas envolvendo aspectos bucais, sem desvios de assunto referentes às outras queixas, optouse por excluir da população sujeitos que pudessem proporcionar dados enviesados. Desta maneira, o estudo trabalhou com uma amostra não-probabilística e intencional. Necessitou-se então de um adequado recorte do objeto da pesquisa e, assim, foram excluídos $\mathrm{da}$ população idosos demenciados, doentes crônicos dependentes fisicamente, idosos que por aspectos circunstanciais no período da coleta não foram localizados, bem como aqueles idosos que não consentiram ou não tiveram a permissão de familiares para participar do estudo. Totalizou-se uma amostra de 261 sujeitos.

Os dados foram coletados através de um questionário, elaborado especificamente para o presente estudo, aplicado em entrevistas domiciliares realizadas pelo próprio pesquisador. O referido questionário estruturado continh ha questões abordando variáveis sociodemográficas e queixas em relação às condições da cavidade bucal. Este instrumento consistiu em um fragmento adaptado de questionário contendo levantamento em saúde bucal em idosos realizado na cidade de Ribeirão Preto/SP (figura 1). É válido ressaltar que o referido instrumento foi elaborado criteriosamente após pré-testes de linguagem e teste piloto, e, portanto, o referido instrumento reflete a validade interna dos resultados. As opções de respostas para a questão envolvendo as queixas foram elaboradas após a saturação e categorização de respostas obtidas com o referido pré-teste.

Os dados, obtidos com as respostas escritas de próprio punho nos questionários, foram digitados em máscara e codificados pelo programa Epidata, para posterior recebimento do tratamento estatístico. Foi realizada análise bivariada, bem como análise descritiva de frequência simples, tendo sido observadas associações entre as variáveis categorizadas de acordo com o teste Qui-quadrado de Pearson e Exato de Fisher. As hipóteses das associações estatísticas foram aceitas frente o valor de $p$ menor ou igual a 0,05 . A análise dos dados foi realizada utilizando-se procedimentos para análise de inquéritos populacionais do programa STATA.

Optou-se por trabalhar com uma população de idosos cadastrados em um serviço de Atenção à Saúde da Família, pela facili- 


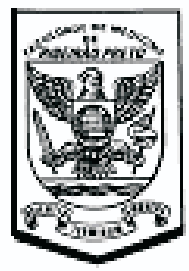

Universidade de São Paulo

Campus de Ribeirão Pre to

Faculdade de Medicina de Ribeirão Preto

Telefone PABX 633-3035 - Telex (0166) 354 - FAX (016) 633-1586 14049-900- RIBEIRAOO PRETO - Est Sào Paub

Instr um ent o da perquisa / fragmento: "queixas bucais relatadas."

Variáveis independentes/ Grupo (1)

1- Idade: $60-69(\quad 70-79() \geq 80($ )

2. Sexo: ( ) M ( ) F

3. Escolaridade: ( ) Primário ( ) Primeiro grau ( ) Segundo grau ( ) Grau universitário

Variáveis exploratórias/ Queixas de ordem geral Grupo (2)

\begin{tabular}{|c|c|c|c|}
\hline 4. Queixas Gerais & Sim & Nă & $\begin{array}{c}\text { Algumas } \\
\text { vezes }\end{array}$ \\
\hline a- Alguma dor na face & ( ) & ( ) & ( ) \\
\hline b. D ificuldade para engolir & ( ) & ( ) & ( ) \\
\hline o. Fadiga mus cular facial ao mastigar & (i) & (i) & (i) \\
\hline d. Sangramento gengival & ( ) & ( ) & ( ) \\
\hline e Mau hálito & () & ( ) & (j) \\
\hline f- Problemas ne ATM & ( ) & ( ) & ( ) \\
\hline g- Boca seca / Xer ostomia & ( ) & ( ) & (j) \\
\hline h. Boca amarga & ( ) & ( ) & ( ) \\
\hline i- Presença de les öes na boca & ( ) & ( ) & ( ) \\
\hline j- Excesso de saliva/ Sialorréia & ( ) & ( ) & ( ) \\
\hline k- Hiper plas ia gengival & ( ) & ( ) & ( ) \\
\hline 1- Bruxis mo & ( ) & ( ) & ( ) \\
\hline m- Diastems & (j) & ( ) & ( ) \\
\hline n-Aparência dos lábios & ( ) & ( ) & ( ) \\
\hline o- Sialolitiase & ( ) & ( ) & ( ) \\
\hline p- Dificuldade para deglutir & ( ) & ( ) & ( ) \\
\hline q. Câimbra mus culares na face & & (1) & (i) \\
\hline
\end{tabular}

Fragmento do instrumento da pesquisa: Saúde bucal em Idosos. Queivas relatadas, Kibeirăo Preto /SP.

Figura 1 - Imagem ilustrativa do questionário utilizado para levantamento de queixas em saúde bucal em idosos. Ribeirão Preto, 2006. 
dade de acesso aos sujeitos da pesquisa e para tentar evitar queixas que poderiam surgir sobre o atendimento odontológico do sistema de saúde público municipal. De acordo com o trabalho de acolhimento, vínculo, escuta, humanização e promoção de saúde do referido Núcleo de Saúde, tais queixas possivelmente não apareceriam. Através dos cadastros dos idosos e informações constantes no Sistema de Informação em Atenção Básica (SIAB), ${ }^{12}$ foram obtidas as informações necessárias para a operacionalização da coleta dos dados. Deste modo, foram levantadas informações sanitárias da área de abrangência do estudo, idade, localização, histórico de saúde e condições fisiológicas dos sujeitos da pesquisa. O mapa da região, contendo área adscrita dividida em microáreas, foi obtido através das agentes comunitárias, o que permitiu um planejamento otimizado para acesso aos idosos.

Fundamentado nesta metodologia, o estudo teve seu projeto aprovado pelo Comitê de Ética do Centro de Saúde Escola da Faculdade de Medicina de Ribeirão Preto da Universidade de São Paulo (CSEFMRP-USP). Todos os idosos, após apresentação da proposta da pesquisa, aceitaram participar da mesma assinando o termo de consentimento livre e esclarecido, atendendo à Resolução no 196/96 do Conselho Nacional de Saúde. ${ }^{13}$

\section{RESULTADOS}

A população de idosos residentes na área de abrangência do NSF III era de 503 indivíduos, segundo informações obtidas junto ao Sistema de Informação em Atenção Básica (SIAB). ${ }^{12}$ Deste total, 242 idosos não participaram do estudo, sendo que apenas 261 , representando $51,8 \%$ do total da população, foram entrevistados (n. 261). Devido a este fato, a tabela 1 traz a descrição das categorias de exclusão desses sujeitos. A maior representatividade para a exclusão dos idosos foi ausência na terceira visita para o sexo masculino $(27,1 \%)$ e recusa para o sexo feminino $(23,4 \%)$. 
Tabela 1 - Distribuição dos motivos de exclusão segundo sexo. Idosos residentes na área de abrangência do Núcleo de Saúde da Família III. Ribeirão Preto, 2006.

\begin{tabular}{|c|c|c|c|c|c|c|c|}
\hline \multirow{2}{*}{\multicolumn{2}{|c|}{ Sexo }} & \multicolumn{2}{|c|}{ Masculino } & \multicolumn{2}{|c|}{ Feminino } & \multicolumn{2}{|c|}{ Sub-total } \\
\hline & & $\mathrm{n}$ & $\%$ & $\mathrm{n}$ & $\%$ & $\mathrm{n}$ & $\%$ \\
\hline \multirow{5}{*}{ 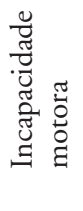 } & Acamado Crônico & 12 & 10,2 & 15 & 12,1 & 27 & 11,2 \\
\hline & AVE / Sequelas & 3 & 2,5 & 3 & 2,4 & 6 & 2,5 \\
\hline & Artrose & 1 & 0,8 & 7 & 5,6 & 8 & 3,3 \\
\hline & Mal de Parkinson & 2 & 1,7 & 3 & 2,4 & 5 & 2,1 \\
\hline & Subtotal & 18 & 15,2 & 28 & 22,5 & 46 & 19,1 \\
\hline \multirow{4}{*}{ 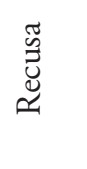 } & Ausência na $3^{a}$ visita & 32 & 27,1 & 10 & 8,1 & 42 & 17,4 \\
\hline & Recusa do idoso & 18 & 15,3 & 29 & 23,4 & 47 & 19,4 \\
\hline & Recusa familiar & 6 & 5,1 & 7 & 5,6 & 13 & 5,4 \\
\hline & Subtotal & 56 & 47,5 & 46 & 37,1 & 102 & 42,2 \\
\hline \multirow{5}{*}{ 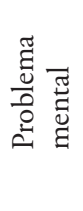 } & Mal de Ausheimer & 1 & 0,8 & 1 & 0,8 & 2 & 0,8 \\
\hline & Demência & 5 & 4,2 & 15 & 12,1 & 20 & 8,3 \\
\hline & Doença mental & 3 & 2,5 & 3 & 2,4 & 6 & 2,5 \\
\hline & Arteriosclerose cerebral & 2 & 1,7 & 3 & 2,4 & 5 & 2,1 \\
\hline & Subtotal & 11 & 9,2 & 22 & 17,7 & 33 & 13,7 \\
\hline \multirow{8}{*}{ 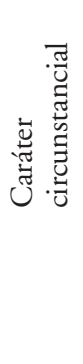 } & Residência sem idoso & 11 & 9,5 & 13 & 10,7 & 24 & 9,7 \\
\hline & Hospitalizado & 6 & 5,1 & 1 & 0,8 & 7 & 2,9 \\
\hline & Cegueira & 2 & 1,7 & 4 & 3,2 & 6 & 2,5 \\
\hline & Surdez & 4 & 3,4 & 5 & 4,0 & 9 & 3,7 \\
\hline & Ca Laringe & 1 & 0,8 & 1 & 0,8 & 2 & 0,8 \\
\hline & Falecido & 9 & 7,6 & 4 & 3,2 & 13 & 5,4 \\
\hline & Subtotal & 33 & 28,1 & 28 & 22,7 & 61 & 25,0 \\
\hline & Total & 118 & 100,0 & 124 & 100,0 & 242 & 100,0 \\
\hline
\end{tabular}

AVE - acidente vascular encefálico

Consideraram-se dois grupos de variáveis: (1) características sociodemográficas; (2) queixas bucais de ordem geral. As características sociodemográficas abordadas foram sexo, idade e escolaridade. A idade foi categorizada em três faixas etárias, 60-69, 70-79 e $\geq 80$. A escolaridade foi trabalhada de maneira clássica, de acordo com levantamentos em saúde bucal de estudos populacionais nacionais. ${ }^{14}$ Quanto às queixas levantadas no presente estudo, abordaram-se toda aquelas envolvendo órgãos e situações da cavidade bucal emergentes no momento da entrevista, exceto as referentes aos dentes e/ 
ou próteses bucais. As queixas de ordem geral em relação à cavidade bucal foram: boca seca; boca amarga; lesões na cavidade bucal; mau hálito; sialorreia (produção excessiva de saliva); dor na face; problemas na articulação temporomandibular (ATM); sangramento gengival; fadiga muscular ao mastigar; aparência dos lábios; diastema; bruxismo; dificuldade para deglutir; hiperplasia gengival e câimbra (dor) musculatura da face.

Na tabela 2 é apresentado um panorama da população de idosos segundo esco- laridade, sexo e idade, onde se observou maior representatividade do sexo feminino $(65,5 \%)$, idade variando entre $60 \mathrm{e}$ 69 anos (46,7\%) e baixa escolaridade. A tabela 3 descreve o diagnóstico da população frente à prevalência das queixas sobre cavidade bucal. Assim, observou-se uma homogeneidade entre as queixas relatadas para ambos os sexos. Cabe ressaltar que a queixa mais prevalente foi a xerostomia/boca seca sendo relatada por $14,4 \%$ dos homens e $14,5 \%$ pelas mulheres entrevistadas.

Tabela 2 - Distribuição da população de idosos segundo escolaridade, sexo e idade. Área de abrangência do Núcleo de Saúde da Família III. Ribeirão Preto/SP, 2006.

\begin{tabular}{|c|c|c|c|c|c|c|c|c|}
\hline \multirow[b]{2}{*}{ Escolaridade } & \multicolumn{2}{|c|}{60 a 69} & \multicolumn{2}{|c|}{70 a 79} & \multicolumn{2}{|c|}{80 e +} & \multicolumn{2}{|c|}{ Total } \\
\hline & $\mathrm{n}$ & $\%$ & $\mathrm{n}$ & $\%$ & $\mathrm{n}$ & $\%$ & $\mathrm{n}$ & $\%$ \\
\hline \multicolumn{9}{|l|}{ Masculino } \\
\hline$<4$ anos & 27 & 72,9 & 22 & 53,6 & 11 & 91,6 & 60 & 66,6 \\
\hline 5 a 8 anos & 4 & 10,9 & 10 & 24,3 & 0 & 0,0 & 14 & 15,5 \\
\hline 9 a 11 anos & 3 & 8,1 & 6 & 14,6 & 0 & 0,0 & 9 & 10,0 \\
\hline$>11$ anos & 3 & 8,1 & 3 & 7,5 & 1 & 8,4 & 7 & 7,9 \\
\hline Subtotal & 37 & 100,0 & 41 & 100,0 & 12 & 100,0 & 90 & 100,0 \\
\hline \multicolumn{9}{|l|}{ Feminino } \\
\hline$<4$ anos & 57 & 67,0 & 53 & 74,6 & 14 & 93,3 & 124 & 72,5 \\
\hline 5 a 8 anos & 12 & 14,1 & 11 & 15,4 & 1 & 6,7 & 24 & 14,0 \\
\hline 9 a 11 anos & 9 & 10,5 & 5 & 7,0 & 0 & 0,0 & 14 & 8,1 \\
\hline$>11$ anos & 7 & 8,4 & 2 & 3,0 & 0 & 0,0 & 9 & 5,4 \\
\hline Subtotal & 85 & 100,0 & 71 & 100,0 & 15 & 100,0 & 171 & 100,0 \\
\hline
\end{tabular}


Tabela 3 - Frequência das queixas sobre cavidade bucal para idosos. Área de abrangência do Núcleo de Saúde da Família III, Ribeirão Preto/SP, 2006.

\begin{tabular}{|c|c|c|c|c|c|c|c|c|}
\hline & \multirow{2}{*}{ Queixas } & \multicolumn{2}{|c|}{ Sim } & \multicolumn{2}{|c|}{ Não } & \multicolumn{2}{|c|}{ Algumas Vezes } & \multirow{2}{*}{$\frac{\text { Total }}{\mathrm{n}}$} \\
\hline & & $\mathrm{n}$ & $\%$ & $\mathrm{n}$ & $\%$ & $\mathrm{n}$ & $\%$ & \\
\hline \multirow{16}{*}{ 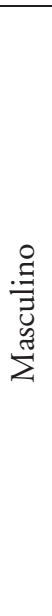 } & Boca seca/ xerostomia & 13 & 14,4 & 65 & 72,2 & 12 & 13,4 & 90 \\
\hline & Mau hálito & 13 & 14,4 & 69 & 76,7 & 8 & 8,9 & 90 \\
\hline & Boca amarga & 5 & 5,6 & 69 & 76,7 & 16 & 17,7 & 90 \\
\hline & Feridas na boca & 13 & 14,4 & 76 & 84,4 & 1 & 1,2 & 90 \\
\hline & ATM & 7 & 7,7 & 77 & 85,5 & 6 & 6,8 & 90 \\
\hline & Dor na face & 5 & 5,6 & 77 & 86,7 & 8 & 7,7 & 90 \\
\hline & Aparência dos lábios & 6 & 6,7 & 83 & 92,2 & 1 & 1,1 & 90 \\
\hline & Aftas & 2 & 2,3 & 84 & 93,3 & 4 & 4,4 & 90 \\
\hline & Fadiga muscular face & 4 & 4,4 & 85 & 94,4 & 1 & 1,2 & 90 \\
\hline & Dificuldade deglutição & 1 & 1,2 & 85 & 94,4 & 4 & 4,4 & 90 \\
\hline & Hiperplasia gengival & 3 & 3,3 & 87 & 96,7 & 0 & 0,0 & 90 \\
\hline & Sialorreia & 3 & 3,3 & 87 & 96,7 & 0 & 0,0 & 90 \\
\hline & Câimbra face & 2 & 2,2 & 88 & 97,8 & 0 & 0,0 & 90 \\
\hline & Sialotitíase & 1 & 1,1 & 89 & 98,9 & 0 & 0,0 & 90 \\
\hline & Diastema & 1 & 1,1 & 89 & 98,9 & 0 & 0,0 & 90 \\
\hline & Bruxismo & 0 & 0,0 & 90 & 100,0 & 0 & 0,0 & 90 \\
\hline \multirow{17}{*}{ 兽 } & Boca seca/ xerostomia & 25 & 14,5 & 112 & 65,5 & 34 & 20,0 & 171 \\
\hline & Boca amarga & 18 & 10,0 & 120 & 70,7 & 33 & 19,3 & 171 \\
\hline & Feridas na boca & 30 & 17,5 & 135 & 78,9 & 6 & 3,6 & 171 \\
\hline & Mau hálito & 22 & 13,0 & 137 & 80,0 & 12 & 7,0 & 171 \\
\hline & ATM & 18 & 10,5 & 142 & 83,0 & 11 & 6,5 & 171 \\
\hline & Dor na face & 16 & 10,0 & 144 & 83,6 & 11 & 6,4 & 171 \\
\hline & Sangramento gengival & 9 & 5,3 & 150 & 87,7 & 12 & 7,0 & 171 \\
\hline & Dificuldade deglutição & 3 & 1,8 & 156 & 91,2 & 12 & 7,0 & 171 \\
\hline & Aftas & 4 & 2,3 & 158 & 92,4 & 9 & 5,3 & 171 \\
\hline & Aparência dos lábios & 11 & 6,4 & 158 & 92,4 & 2 & 1,2 & 171 \\
\hline & Fadiga muscular face & 7 & 4,1 & 161 & 94,1 & 3 & 1,8 & 171 \\
\hline & Hiperplasia gengival & 5 & 2,9 & 166 & 97,1 & 0 & 0,0 & 171 \\
\hline & Câimbra face & 2 & 1,2 & 169 & 98,8 & 0 & 0,0 & 171 \\
\hline & Bruxismo & 2 & 1,2 & 169 & 98,8 & 0 & 0,0 & 171 \\
\hline & Diastema & 1 & 0,6 & 170 & 99,4 & 0 & 0,0 & 171 \\
\hline & Sialorreia & 1 & 0,6 & 170 & 99,4 & 0 & 0,0 & 171 \\
\hline & Sialolitiase & 0 & 0,0 & 171 & 100 & 0 & 0,0 & 171 \\
\hline
\end{tabular}

As tabelas 4 e 5, respectivamente, trazem as distribuições das queixas mais frequentes segundo idade e escolaridade, observando-se possíveis associações estatisticamente significativas. De acordo com tais tabelas, observou- se associação entre as variáveis queixa de mau hálito e escolaridade $(p=0,011)$, sendo que neste caso idosos com menor escolaridade se queixaram de mau hálito em maior proporção que os idosos de elevada escolaridade. 
Tabela 4 - Distribuição percentual das queixas mais frequentes segundo idade. Idosos residentes na área de abrangência do Núcleo de Saúde da Família III. Ribeirão Preto/SP, 2006.

\begin{tabular}{lccccccccc}
\hline & Idade & \multicolumn{2}{c}{$60-69$} & \multicolumn{2}{c}{$70-79$} & & $\geq 80$ & Total & \multirow{2}{*}{$\mathrm{p}$} \\
\cline { 2 - 7 } Queixa sim & $\mathrm{n}$ & $\%$ & $\mathrm{n}$ & $\%$ & $\mathrm{n}$ & $\%$ & $\mathrm{n}$ & \\
\hline Boca seca & 9 & 23,6 & 25 & 65,7 & 4 & 10,7 & 38 & 0,271 \\
Mau hálito & 20 & 57,4 & 13 & 37,1 & 2 & 5,5 & 35 & 0,276 \\
ATM & 14 & 58,3 & 9 & 37,5 & 1 & 4,2 & 24 & 0,221 \\
\hline
\end{tabular}

ATM - Articulação Temporomandibular. * Associação estatisticamente significativa. Valores em negrito - Teste exato de Fisher

Tabela 5 - Distribuição percentual das queixas mais frequentes segundo escolaridade. Idosos residentes na área de abrangência do Núcleo de Saúde da Família III. Ribeirão Preto/ SP, 2006.

\begin{tabular}{|c|c|c|c|c|c|c|c|c|c|c|}
\hline \multirow{2}{*}{ Queixa sim } & \multicolumn{2}{|c|}{$\leq 4$} & \multicolumn{2}{|c|}{$5-8$} & \multicolumn{2}{|c|}{$9-11$} & \multicolumn{2}{|c|}{$\geq 11$} & \multirow{2}{*}{$\begin{array}{c}\text { Total } \\
\mathrm{n} \\
\end{array}$} & \multirow[t]{2}{*}{$\mathrm{p}$} \\
\hline & $\mathrm{n}$ & $\%$ & $\mathrm{n}$ & $\%$ & $\mathrm{n}$ & $\%$ & $\mathrm{n}$ & $\%$ & & \\
\hline Boca seca & 23 & 60,5 & 5 & 13,1 & 7 & 18,4 & 3 & 8,0 & 38 & 0,133 \\
\hline Mau hálito & 26 & 74,2 & 4 & 11,4 & 2 & 5,8 & 3 & 8,6 & 35 & $0,011^{*}$ \\
\hline ATM & 13 & 54,1 & 7 & 29,3 & 2 & 8,3 & 2 & 8,3 & 24 & 0,401 \\
\hline
\end{tabular}

ATM - Articulação temporomandibular.

*Associação estatisticamente significativa. Valores em negrito - Teste exato de Fisher

\section{DISCUSSÃO}

De acordo com a tabela 1 , a primeira categoria de exclusão foi representada por indivíduos que, frente a situações fisiológicas e/ou patológicas previamente observadas no histórico de saúde do paciente, não seriam capazes de participar de uma entrevista. Assim, excluíram-se idosos acamados, aqueles que sofreram algum acidente vascular cerebral (AVC) e os por- tadores de limitações decorrentes de artrose e mal de Parkinson. Estes casos foram excluídos quando apresentavam sequelas motoras graves que impossibilitavam atividades simples da vida diária. Esses idosos não foram entrevistados, pois poderiam se queixar de problemas com o autocuidado e problemas bucais causados por sequelas das referidas patologias. Esta categoria representou 19,1\% dos não-participantes (tabela 1). 
Uma segunda categoria foi a exclusão por decisão pessoal do idoso e/ou família, caracterizada como recusa verbal, bem como o não-recebimento do entrevistador na residência. Nesta categoria foram agrupados casos de recusa do próprio idoso, recusa da família e ausência do idoso no domicílio na terceira visita agendada. O núcleo de saúde da família em questão era composto por uma população onde 19,4\% (47) idosos não consentiram com as entrevistas e 5,4\% (13) não obtiveram autorização de familiares para participar do estudo.

Os idosos também foram selecionados de acordo com a preservação de sua capacidade mental; assim, possíveis erros relacionados à confiabilidade das respostas foram evitados. Incluíram-se nesta categoria indivíduos que tivessem qualquer problema de ordem mental. Foram agrupados, desta maneira, idosos com mal de Alzheimer, portadores de qualquer doença mental e arterosclerose cerebral, afecções estas constantes nos prontuários, bem como aqueles idosos que, submetidos ao Mini-Exame do Estado Mental, ${ }^{15,16}$ não obtiveram a pontuação mínima caracterizando demência. Do total de idosos representados por esta categoria, a maior prevalência foi de demência $(8,3 \%)$ (tabela 1).

A última categoria, nomeada de caráter circunstancial, esteve relacionada às situações vividas pelo entrevistador no trabalho de campo. Diferentes situações foram enfrentadas durante as visitas domiciliares, tendo sido observados os seguintes motivos de exclusão: idosos falecidos, residência sem morador idoso, idosos hospitalizados no período de coleta de dados, idosos cegos/ diminuição da acuidade visual, idosos surdos e idosos com câncer de laringe e/ou cordas vocais. A tabela 1 mostra a distribuição das categorias assinaladas.

Ao se analisar mais profundamente cada motivo de exclusão segundo sexo, observouse que mais indivíduos do sexo masculino $(27,1 \%)$ foram excluídos por não terem sido encontrados no domicílio no momento da entrevista, na terceira visita. Acredita-se que, devido aos afazeres domésticos e familiares, muitas vezes como cuidadores, foi mais fácil o acesso do pesquisador às mulheres; sendo assim, apenas $8,1 \%$ não foram encontradas seguindo o proposto. Entretanto, um dado interessante também relacionado ao sexo feminino foi a maior recusa por decisão pessoal $(23,4 \%)$ em relação aos homens $(15,3 \%)$ (tabela 1). Esta alta taxa de recusa em estudos com idosos também foi encontrada na literatura. ${ }^{7,17}$

Do total de idosos excluídos, $5,4 \%$, representados por 13 indivíduos, não contaram com a autorização de familiares (filhos, netos, genros, noras etc.) para participar do estudo (tabela 1), o que evidencia a falta de autonomia dos idosos em algumas famílias, visto que o entrevistador, nestes casos, não teve acesso ao idoso. Dentre os motivos da quarta categoria, especial atenção foi dada ao motivo referente à residência sem morador idoso. Neste caso, 24 (9,7\%) indivíduos estavam cadastrados em residências que no período da coleta de dados estavam abandonadas, com placas de disponibilidade para 
locação imobiliária, e casos onde atuais moradores relataram que o idoso, procurado pelo pesquisador, estava morando em "residências de outros parentes" ou em "casas de repouso" (tabela 1).

Em nossa sociedade, algumas famílias não estão preparadas para conviver com o indivíduo idoso, e este raciocínio leva à institucionalização do indivíduo de idade avançada. ${ }^{18}$ Somando-se todos esses aspectos, para participação na pesquisa, o idoso necessitava dos sentidos da audição, visão e fala, sendo que, de acordo com tais requisitos, seis indivíduos cegos e/ ou com acuidade visual comprometida, nove indivíduos surdos, um com câncer nas cordas vocais e outro com câncer na laringe, foram excluídos da pesquisa (tabela 1).

Ao se discutir sobre os idosos que participaram ativamente da pesquisa respondendo ao questionário, tem-se que do total desta população $171(65,5 \%)$ eram do sexo feminino e 90 (34,5\%) do sexo masculino. A idade dos idosos entrevistados variou de 60 a 93 anos, sendo a média de 71,8 anos e moda igual a 68 anos para o sexo masculino, e 70,1 e moda igual a 64 para o sexo feminino. Do total, $46,7 \%$ tinham idade entre 60 e 69 anos, $43,3 \%$ com idade entre 70 e 79 anos e apenas 10,0\% com 80 anos ou mais. A maior frequência de mulheres idosas, no presente estudo, se assemelha a outros estudos na literatura. ${ }^{19,20}$ A grande maioria da população apresentou baixa escolaridade (tabela 2). Este aspecto, bem como a existência de um percentual de analfabetismo, era previsível, pois se estudou uma população brasileira com mais de 60 anos de idade - portanto, indivíduos que na idade em que deveriam ingressar na escola, o acesso ao conhecimento e a educação eram mais restritos que nos dias atuais. Neste caso, a baixa escolaridade foi mais frequente entre pessoas mais velhas à semelhança da literatura estudada. ${ }^{14}$

Todos os idosos entrevistados relataram alguma queixa em relação à condição da saúde bucal, e este fato se distancia dos dados encontrados na literatura, ${ }^{7}$ onde poucos idosos se queixaram da condição da cavidade bucal. A abordagem das queixas foi feita tendo como possíveis respostas "sim"; "não" e "algumas vezes". O fato de se possibilitar as respostas "algumas vezes" apenas teve o objetivo de mostrar de maneira descritiva o aspecto sazonal de algumas queixas. As associações foram feitas frente às queixas mais prevalentes no aspecto positivo (respostas "sim"), as quais refletiam problemas frequentes na vida do idoso. No total foram levantadas 17 queixas com prevalências semelhantes para os sexos, como mostra a tabela 3. Foi possível observar uma diversidade de queixas envolvendo a cavidade bucal. Assim, observaram-se queixas relacionadas às glândulas salivares, problemas musculares e articulares.

Esse fato mostrou que, na presente população, existiam queixas de grande amplitude que não envolviam dentes e/ou uso de próteses. Fato interessante esteve relacionado a queixas como dor na cavidade bucal (sem ser de etiologia dentária), boca amarga e queixas de origem estética. Observou-se a 
baixa prevalência da queixa "alguma dor", para ambos os sexos (10\% sexo feminino e 5,6\% sexo masculino) (tabela 3). Acredita-se que este fato esteve associado ao limiar de dor, pois a sensibilidade dolorosa diminui com a idade, fazendo com que a dor não seja uma queixa frequente para o idoso. ${ }^{21}$ Outra variedade de queixas foram as queixas envolvendo aspectos estéticos, que refletiam a preocupação com a aparência dos lábios (6,4\% das mulheres e 6,7\% dos homens) bem como a aparência dos dentes (diastemas) (1,1\% nas mulheres e $0,6 \%$ nos homens) (tabela 3). Apesar de poucos relatos, os idosos da referida população também se queixaram da aparência que mostram para o mundo social. As mulheres idosas queixaram-se mais frequentemente com o passar da idade, fato este que não ocorreu no sexo masculino. Estudo com população de idosos independentes observou que idade e gênero têm pouca influência na saúde bucal. ${ }^{22}$

As queixas mais prevalentes foram boca seca, problemas de ATM e mau hálito. Neste contexto, as associações estatísticas foram direcionadas partindo-se das variáveis independentes idade e escolaridade. Deste modo, observaram-se possíveis dependências para as três queixas (sim) citadas anteriormente. Optou-se por buscar associações com a variável escolaridade, pois, como observado na literatura, quanto maior a escolaridade, maior são os relatos de queixas em relação às condições da cavidade bucal. ${ }^{23}$ No decorrer do texto discutir-se-ão tais aspectos.

A queixa de boca seca esteve presente em $14,4 \%$ das mulheres e $14,5 \%$ dos homens, como mostra a tabela 3. Pôde-se afirmar que esta queixa foi também frequentemente relatada de maneira sazonal, ou seja, acreditase que outros fatores, como o uso de medicamentos ou patologias de glândulas salivares ,poderiam estar associadas a esta queixa. Estudos mostram que $55,0 \%$ dos idosos se queixaram de boca seca, e este valor foi superior aos dados do presente estudo. ${ }^{24} \mathrm{Ou}$ tras investigações assinalaram alta prevalência de queixa de boca seca em idosos. ${ }^{17,25-28}$ A queixa de boca seca está associada à quantidade de dentes, visto que a ausência dos dentes pode proporcionar baixa secreção salivar. ${ }^{29}$ No presente estudo, não foram observadas associações estatisticamente significantes entre queixas de boca seca e idade $(p=0,271)$ e escolaridade $(p=0,133)$ como mostram as tabelas 4 e 5 . A associação entre idade e queixa de boca seca foi observada na literatura em estudo populacional que relatou que essa queixa foi maior em sujeitos com idades mais avançadas $(p=0,015) .{ }^{30}$ Outro estudo com sujeitos idosos mostrou que a queixa de dificuldade mastigatória, devido à baixa secreção salivar, não esteve associada à idade. ${ }^{31}$

O número de pessoas se queixando de halitose vem aumentando, sendo que de 6,0 a $23,0 \%$ da população mundial possuem halitose, independentemente da situação socioeconômica. ${ }^{32} \mathrm{O}$ mau hálito foi relatado como queixa em $14,4 \%$ dos homens e $13,9 \%$ das mulheres (tabela 3 ), sendo que essa queixa diminuiu com a idade. Acredita-se que esta baixa prevalência pode estar relacionada com a não-percepção deste sinal na população estudada. 
Queixar-se de halitose está diretamente relacionada com autopercepção. A autopercepção é fundamental para se estudar halitose, ${ }^{33}$ e estudo semelhante ao presente observou que muitos sujeitos se queixavam de halitose; no entanto, mais da metade destes quase não tinham mau hálito. ${ }^{19}$ Como mostram as tabelas 4 e 5 , não houve associação estatisticamente significativa entre queixa de mau hálito e idade $(p=0,276)$, mas houve associação entre queixa de mau hálito e escolaridade $(p=0,015)$. Acredita-se que quanto mais elevado o nível educacional do sujeito, maior é a autopercepção e, consequentemente, maiores são os relatos desta queixa. No presente estudo, idosos com baixa escolaridade se queixaram menos, proporcionalmente, de halitose, fato que se assemelha à literatura. ${ }^{33}$ Paralelamente a este, observou-se na literatura que homens se queixaram mais de halitose e que essa queixa aumentou com a idade. ${ }^{34}$

As queixas referentes aos problemas de ATM envolviam aspectos como estalido na região do ouvido, desconforto na região da articulação ao movimentar a mandíbula e dores na articulação em questão. Esta foi relatada por $7,7 \%$ dos homens e $10,0 \%$ das mulheres (tabela 3), e não esteve associada à idade e à escolaridade. $\mathrm{Na}$ literatura não foram encontrados estudos com metodologia semelhante, mas se observou que queixas envolvendo a ATM estavam relacionadas a relatos de dores de ouvido, cansaço muscular ao mastigar e ruídos na região do ouvido, percepções observadas no presente estudo. ${ }^{35}$

\section{CONSIDERAÇÕES FINAIS}

O presente estudo contém algumas características que se mostraram importantes para o desenvolvimento, planejamento e execução de estudos com populações de idosos. Ao estudar queixas envolvendo aspectos da saúde desses indivíduos, foi possível observar que se deve adotar uma adequada seleção dos sujeitos para um adequado recorte do objeto da pesquisa, pois muitos são os motivos de exclusão de idosos em inquéritos domiciliares.

Aspectos de saúde até aspectos relacionados à relação familiar dificultaram o acesso às entrevistas, o que gerou certa perda de sujeitos na população de referência. Com isso, a categorização dos motivos de exclusão possibilitou coerência e validade interna dos $\mathrm{da}$ dos. Tais exclusões devem ser levadas em conta quando se estudam idosos em inquéritos domiciliares. Este foi um estudo de grande valia local, porém pode ser utilizado como exemplo para outros estudos semelhantes. Os resultados permitiram o conhecimento local quanto aos aspectos estudados, e permitirão trabalhos na promoção de saúde, bem como a análise de necessidades em saúde bucal para os referidos idosos.

Quando observadas as frequências das queixas, não foram verificadas diferenças significativas entre os sexos. Foi possível observar que os idosos têm diferentes visões e valores em relação à saúde bucal, pois diversas e diferentes queixas puderam ser detectadas. Seguindo-se tal racio- 
cínio, o presente estudo suscitou a importância de se estudar outras variáveis em relação ao tema e à população idosa. Estudos com queixas de saúde bucal abordando pessoas idosas é assunto emergente na saúde pública, e tal fato dificultou a busca por pesquisas que resgatassem esse assunto para compor o grupo dos estudos referenciados.

As queixas observadas neste estudo sugerem que profissionais da saúde bucal devem se preocupar com outros órgãos da cavidade oral e não só os dentes, visto que

\section{REFERÊNCIAS}

1. Instituto Brasileiro de Geografia e Estatística. Censo demográfico: Brasil, 1991. Rio de Janeiro: IBGE. Disponível em: URL: http: www.ibge.gov.br.

2. Nadanovsky P. O aumento da produção científica odontológica brasileira na saúde pública. Cad Saude Publica 2006; 22(5): Editorial.

3. Veras R. Em busca de uma assistência adequada à saúde do idoso: revisão de literatura e aplicação de um instrumento de detecção precoce e de previsibilidade de agravos. Cad Saude Publica 2003; 19(3): 705-15.

4. Brasil. Política Nacional de Saúde Bucal, Diretrizes 2004. Diretrizes da Política Nacional de Saúde Bucal. Brasília: Ministério da Saúde, Secretaria de Atenção à Saúde; 2004. 16p.

5. Mishima SM, Pereira MJB, Matsumoto S, Nascimento MAA, Fortuna CM, Teixeira RA. O desafio do cuidar em saúde coletiva. In: Anais do $4^{\circ}$ Fórum Mineiro
$100 \%$ dos idosos entrevistados relataram alguma queixa envolvendo órgãos da cavidade bucal e que tais queixas não estiveram associadas ao sexo. Ficou claro que alguns idosos também se preocuparam com aspectos estéticos evidenciados pelas queixas referentes aos lábios e aos diastemas. Observou-se, ainda, que muitas queixas foram momentâneas, possivelmente originadas pelo uso de certos medicamentos e patologias bucais, dentre outras situações. Frente a estas considerações, o presente estudo suscita novas discussões frente à temática da saúde bucal e do idoso.

de Enfermagem; 1994; Uberlândia. Belo Horizonte, 1994. p. 334-5.

6. Ramos DD, Lima MADS. Acesso e acolhimento aos usuários em uma unidade de saúde de Porto Alegre, Rio Grande do Sul, Brasil. Cad Saude Publica 2003; 19(1): 27-34.

7. Hiramatsu DA, Franco LJ, Tomita NE. Influência da aculturação na autopercepção dos idosos quanto à saúde bucal em uma população e origem japonesa. Cad Saude Publica 2006; 22(11): 2441-8.

8. Meira RLC, Gordilho ACB, Papaleo Neto M, Nery MR. Sinais e sintomas em gastroenterologia geriátrica. In: Guimarães RM, Cunha UGV. Sinais e sintomas em geriatria. São Paulo: Revinter; 1989. p.113-30.

9. Orellana MF, Lagravère MO, Boychuk DGJ, Major PW, Flores-Mir C. Prevalence of xerostomia in population-based samples: a systematic review. J Public Health Dent 2006; 66 (2): 152-8. 
10. Madeira AA, Madeira L. O paciente geriátrico e a complexidade de seu tratamento. Rev Bras Odontol 2000; 57(6): 350-1.

11. Ship JA. Oral health in the elderly. What's missing? Oral Surg Oral Med Oral Pathol Oral Radiol Endod2004; 98(6): 625-6.

12. Brasil. Ministério da Saúde. Departamento de Atenção Básica. Manual do Sistema de Informação de Atenção Básica(SIAB). 2003. Disponível em: URL: dtr2004.saude.gov.br/dab/docs/ publicacoes/manual_siab2000.pdf

13. Brasil. Ministério da Saúde. Resolução 196/96 do Conselho Nacional de Saúde/ MS sobre Diretrizes e Normas Reguladoras de Pesquisa Envolvendo seres humanos. Diário Oficial da União, 10 de outubro de 1996.

14. Monti LM, Justi MM, Farjado RS, Zavanelli AC. Análise comparada da saúde bucal do idoso na cidade de Araçatuba. Revista brasileira de geriatria e gerontologia 2006; 9(2): 35-47.

15. Folstein MF, Folstein SE. A practical method for granding the cognitive state of patients for the clinician. J Psychiatr Res 1975; 12: 189-98.

16. Seabra MLV, Concílio GV, Villares JB, Carlini EA. Avaliação do teste "MiniMental State" em voluntários e pacientes brasileiros. Rev Bras Psiquiatr 1990; 12(1-4): 1-7.

17. Carvalho IMM. Avaliação sócioodontológica de 300 pessoas idosas de Bauru -SP. [tese]. Bauru: Faculdade de Odontologia, Universidade de São Paulo; 2000.

18. Boechat NS. Asilamento uma visão. In: . Caminhos do envelhecer. Rio de Janeiro: SBGG, Revinter; 1994. p. 199-205.
19. Oho T, Yoshida Y, Shimazaky Y, Ymashita Y, Koga T. Psycholgical condition of patients complaining of halitosis. J Dent 2001; 29: 31-33.

20. Caldas Júnior AF, Figueredo ACL, Soriano EP, Sousa EHA, Melo JBG, Vilela AS. Prevalência de cárie e edentulismo em idosos de Recife -Pernambuco- Brasil. Revista brasileira de ciências da saúde 2002; 6(2): 113-22.

21. Telford CW, Sawrey JM. O indivíduo excepcional. Rio de Janeiro: Zahar; 1984.

22. Macentee MI, Stolar E, Click N. Influence old age and gender on oral health and related behavior in an independent elderly population. Community Dent Oral Epidemiol 1993; 21(4): 234-9.

23. Mojon P, Macentee MI. Discrepancy between need for prosthodontic treatment and complaints in an elderly edentulous population. Community Dent Oral Epidemiol 1992; 20(1): 48-52.

24. Jales MA, Cabral RR, Silva HJ, Cunha DA. Caracteristicas do sistema estmatognático em idosos: diferenças entre instituição pública e privada. Rev CEFAC 2005; 7(2): 178-87.

25. Oliveira JA, Ribeiro EDP, Bonachela WC, Capelozza ALA. Perfil do paciente odontogeriatrico da faculdade de odontologia de Bauru-USP. Revista brasileira de prótese clínica \& laboratorial : PCL 2002; 4(17): 71-9.

26. Salles AE. Estudo populacional relacionando saúde geral, uso de medicamentos, condutas, hábitos e tempo de uso de prótese total em idosos desdentados totais na cidade de Ribeirão Preto. [dissertação]. Ribeirão Preto: Faculdade de Odontologia, Universidade de São Paulo; 2002.

27. Silva VCC. Avaliação bucal e nutricional em pacientes senescentes. [dissertação]. 
São Paulo: Faculdade de Odontologia, Universidade de São Paulo; 2005.

28. Kruger E, Tennat M, Smith K, Peachey J. The oral health and treatment needs of ommunity-dwelling older people in a rural town in Western Austalia. Australian Journal On Aging 2007; 26(1): 15-20.

29. Bergdahl, M. Salivary flow and oral complaints in adult dental patients. Community Dent Oral Epidemiol 2000; 28(1): 59-66.

30. Bretz WA, Loesche WJ, Chen YM, Schork MA, Dominguez BL, Grosman N, et al. Minor salivary gland secretion in the elderly. Oral Surg Oral Med Oral Pathol Oral Radiol Endod 2000; 89(6): 696-701.

31. Ow PKK, Loh T, Neo J, Khoo J. Perceived masticatory funtion among people. J Oral Rehabil 1997; 24: 131-7.
32. Miyazaki H, Sakao S, Katoh Y, Takehara T. Correlation between volatile sulphur compounds and certain oral health measurements in general population. J Periodontol 1995; 66: 679-84.

33. Al-Ansari J, Boodai H, Al-Sumait N, AlKhabbaz AK, Al-Shammari KF, Salako N. Factors associated with self-reported halitosis in Kwaiti patients. J Dent 2006; 34: 444-9.

34. Nadanovsky P, Carvalho LBM, Ponde de Leon A. Oral malodour and its association with age and sex in a general population in Brazil. Oral Dis 2007; 13: 105-9.

35. Ribeiro RA, Mollo Júnior FA, Arioli Júnior JN, Pinelli LAP. Sintomas de disfunção craniomandibular. Rev Gaucha Odontol 2003; 51(2): 123-6. 


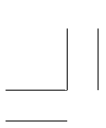

